\title{
Large thermoelectric power variations in epitaxial thin films of layered perovskite GdBaC02O5.5 \pm with a different preferred orientation and strain
}

Chatterjee, Arindom; Chavez-Angel, Emigdio; Ballesteros, Belén; Caicedo, José Manuel; Padilla-Pantoja, Jessica; Leborán, Victor; Sotomayor Torres, Clivia M.; Rivadulla, Francisco; Santiso, José

Published in:

Journal of Materials Chemistry A

Link to article, DOI:

$10.1039 / \mathrm{d} 0 \mathrm{ta} 04781 \mathrm{c}$

Publication date:

2020

Document Version

Peer reviewed version

Link back to DTU Orbit

Citation $(A P A)$ :

Chatterjee, A., Chavez-Angel, E., Ballesteros, B., Caicedo, J. M., Padilla-Pantoja, J., Leborán, V., Sotomayor Torres, C. M., Rivadulla, F., \& Santiso, J. (2020). Large thermoelectric power variations in epitaxial thin films of layered perovskite $\mathrm{GdBaCo} \mathrm{O}_{5}$ with a different preferred orientation and strain. Journal of Materials Chemistry A, 8(38), 19975- F9983. https://doi.org/10.1039/d0ta04781c

\section{General rights}

Copyright and moral rights for the publications made accessible in the public portal are retained by the authors and/or other copyright owners and it is a condition of accessing publications that users recognise and abide by the legal requirements associated with these rights.

- Users may download and print one copy of any publication from the public portal for the purpose of private study or research.

- You may not further distribute the material or use it for any profit-making activity or commercial gain

- You may freely distribute the URL identifying the publication in the public portal 
This is the accepted version of the article:

Chatterjee A., Chavez-Angel E., Ballesteros B., Caicedo J.M., Padilla-Pantoja J., Leborán V., Sotomayor Torres C.M., Rivadulla F., Santiso J.. Large thermoelectric power variations in epitaxial

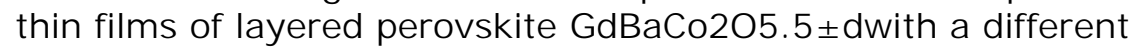
preferred orientation and strain. J ournal of Materials Chemistry A, (2020). 8. : 19975 - . 10.1039/d0ta04781c.

Available at: https://dx.doi.org/10.1039/d0ta04781c 


\section{Large thermoelectric power variations in epitaxial thin films of layered perovskite $\mathrm{GdBaCo}_{2} \mathrm{O}_{5.5 \pm \delta}$ with different preferred orientation and strain}

Arindom Chatterjeeף, ¥, *, Emigdio Chavez-Angelף, Belén Ballesteros", José Manuel Caicedo",

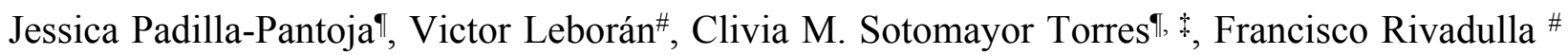
and José Santiso*, $q$

I Catalan Institute of Nanoscience and Nanotechnology (ICN2); CSIC and Barcelona Institute of Science and Technology (BIST), Bellaterra-08193, Spain

$¥$ Current address of the author: Department of Energy Conversion and Storage, Technical University of Denmark, DK-2800 Kgs Lyngby, Denmark

\# Centro Singular de Investigación en Química Biolóxica e Materiais Moleculares (CIQUS), Departamento de Química-Física, Universidade de Santiago de Compostela, Santiago de Compostela 15782, Spain

\$ ICREA-Institució Catalana de Recerca i Estudis Avançats, 08010 Barcelona, Spain

*Corresponding authors

JS (jose.santiso@icn2.cat)

AC (arichatterjee1990@gmail.com)

\section{Abstract:}

This work describes the growth of thin epitaxial films of the layered perovskite material $\mathrm{GdBaCo}_{2} \mathrm{O}_{5.5 \pm \delta}$ (GBCO) on different single crystal substrates $\mathrm{SrTiO}_{3}(\mathrm{STO}),\left(\mathrm{LaAlO}_{3}\right)_{0.3}\left(\mathrm{Sr}_{2} \mathrm{TaAlO}_{6}\right)_{0.7}(\mathrm{LSAT})$ and $\mathrm{LaAlO}_{3}$ (LAO) as a way to study changes in the thermoelectric properties by means of the induced epitaxial strain. In addition to strain changes the films grow with considerably different preferred orientations and domain microstructure: GBCO films on STO are purely $c$-axis oriented $\left(c_{\perp}\right)$ with an average $0.18 \%$ in-plane tensile strain; GBCO on LSAT are composed of domains with mixed orientation $\left(c_{\|}\right.$and $\left.c_{\perp}\right)$ with an average $0.71 \%$ in-plane compressive strain; while on LAO it is $b$-axis oriented $\left(c_{\|}\right)$with an average $0.89 \%$ in-plane compressive strain. These differences result in important cell volume changes, as well as in the orthorhombicity of $a-b$ plane of GBCO structure, which in turns induce a change in the sign and temperature dependence of the thermopower, while the electrical conductivity remains almost unchanged. In general, compressively strained films show negative $S$ thermopower ( $n$-type) while tensile strained film show positive $S$ ( $p$-type) at low temperatures, probing the adaptive nature of $\mathrm{GdBaCo}_{2} \mathrm{O}_{5.5 \pm \delta}$ compound. These 
results point to the spontaneous generation of oxygen vacancies to partially accommodate the epitaxial stress as the main cause for this effect.

\section{Introduction}

Thermoelectric Seebeck effect is very important from the perspective of future clean energy utilization and management, as it is a fundamental factor in the performance of solid-state thermoelectric generators (TEGs). Efficiency of such devices is related to the thermoelectric figure of merit; zT=(T.S $/ \rho . \kappa)$, where $S$, $\rho, \kappa$ and $T$ are Seebeck coefficient, electronic resistivity, thermal conductivity and mean temperature, respectively ${ }^{1}$.

Quest for $p$ - and $n$-type high performance thermoelectric materials, both necessary for implementation in TEG modules, remains an active field of research. ${ }^{2-3}$ In particular, focus has been turned into the oxide materials because of their excellent chemical stability at high temperatures, high abundance and environmental friendliness of the constitute elements. ${ }^{3}$ However, just a few oxides have shown promising thermoelectric properties with sufficient stability and zT values close to unity at high temperature. Examples include $\mathrm{Na}_{\mathrm{x}} \mathrm{CoO}_{2},{ }^{4,5}$ misfit layer cobaltates $\mathrm{Bi}_{2} \mathrm{Sr}_{2} \mathrm{Co}_{2} \mathrm{O}_{\mathrm{y}}{ }^{6,7}$ and $\mathrm{Ca}_{3} \mathrm{Co}_{4} \mathrm{O}_{9},{ }^{8}$ which are $p$-type, and $\mathrm{ZnO},{ }^{9} \mathrm{Nb} / \mathrm{La}$ doped $\mathrm{SrTiO}_{3},{ }^{10,11}$ or $\mathrm{CaMnO}_{3},{ }^{12}$ which are $n$-type. Within the cobalt oxide-based family of compounds the understanding of the relationship between complex spin and orbital degeneracy of cobalt ions ${ }^{13-14}$ and the large $S$ of $p$-type layered misfit cobaltates remains a challenging research topic. ${ }^{15-17}$

In this regard, layered double perovskite $\mathrm{GdBaCo}_{2} \mathrm{O}_{5.5 \pm \delta}(\mathrm{GBCO})$ compound shows some characteristics that make it particularly interesting from the thermoelectric point of view: i) it shows a change of the sign of Seebeck coefficient from negative to positive (i.e., from $n$ - to $p$-type), just by increasing/decreasing the oxygen content ${ }^{18,19}$ at both sides of $\delta=0$ in $\mathrm{GdBaCo}_{2} \mathrm{O}_{5.5 \pm \delta}$ (stability range from $0.5<\delta<+0.3)$; ii) the origin of the high positive and negative Seebeck coefficient was proposed from the contribution of spin and orbital degrees of freedom (i.e., configurational entropy) of Co ions, which differs significantly from conventional electronic band structure engineering; ${ }^{18,20}$ and iii) the layered crystal structure and oxygen vacancy ordering resulted in a remarkable uniaxial anisotropy of $\mathrm{Co}^{3+} \mathrm{spins}^{21}$, which could, in principle, bring about highly anisotropic thermoelectric properties.

In this report, we show that epitaxial engineering can be used to promote the growth of high crystal quality $\mathrm{GdBaCo}_{2} \mathrm{O}_{5.5}$ with different crystal orientations and strain, and hence to study their effect in the thermoelectric properties of this material.

The crystal structure of $\mathrm{GdBaCo}_{2} \mathrm{O}_{5.5}$ is orthorhombic Pmmm at room temperature ${ }^{22}$, with lattice parameters $a=3.862, b / 2=3.934$ and $c / 2=3.786 \AA$. In order to induce different film substrate mismatch 
for this study we have selected the following substrates: (001) $\mathrm{SrTiO}_{3}(\mathrm{STO})$, $\left(\mathrm{LaAlO}_{3}\right)_{0.3}\left(\mathrm{Sr}_{2} \mathrm{TaAlO}_{6}\right)_{0.7}$ (LSAT) and $\mathrm{LaAlO}_{3}$ (LAO), with pseudocubic lattice parameters 3.905, 3.870 and $3.790 \AA$, respectively.

\section{Experimental methods}

Epitaxial films with 25 to $40 \mathrm{~nm}$ thickness were grown by pulsed laser deposition (PLD) technique on STO, LSAT and LAO substrates. Preliminary study indicated that in these range of thickness films grow fully strained with the substrates and no substantial relaxation takes place. The substrate temperature during film deposition was kept constant to $850{ }^{\circ} \mathrm{C}$ with a laser fluency of $\sim 1.5 \mathrm{~J} / \mathrm{cm}^{2}$ and a repetition rate of $10 \mathrm{~Hz}$. The oxygen partial pressure during the growth and heating-cooling steps was 60 mTorr.

The structural characterizations of the as grown films were carried out by standard X-ray diffraction in

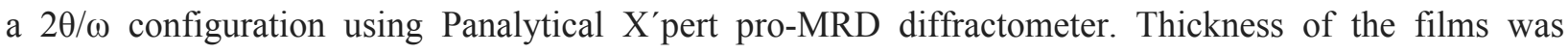
determined from the X-ray reflectivity (XRR) technique. The in-plane and the out-of-plane lattice constants of the films were accurately calculated from the X-ray reciprocal space maps (RSMs). Microstructural characterization of the films were carried out by high-resolution transmission electron microscopy (HRTEM) using a FEI Tecnai G2 F20 microscope with a field emission gun working at $200 \mathrm{kV}$. Electron Energy-Loss Spectroscopy (EELS) experiments were performed in STEM mode at $200 \mathrm{kV}$ using a Gatan Quantum SE 963 Imaging Filter (GIF). Energy-dispersive x-ray (EDX) spectroscopy experiments were performed using an EDAX super ultra-thin window (SUTW) X-ray detector.

Electrical conductivity measurements were carried out in a Van der Pauw configuration from 50 to 300 K. For Seebeck coefficient measurements, a Pt heater and two Pt resistors/thermometers were patterned on the top of thin film surface by optical lithography. Details of the thermopower experiments are presented in Fig. S1 (in Electronic Supplementary Information, ESI). The Pt line heater was electrically isolated from the rest of the film in order to avoid any current leakage from the heater to the film. Similar metal electrodes were patterned on a bare STO substrate to check whether there could be any contribution of STO substrates to the thermopower of the GBCO thin films. Prior to the lithography processes, the STO substrates were treated with exactly the same conditions as GBCO films (same temperature and $p \mathrm{O}_{2}$ atmosphere). The STO substrate was still electrically insulating after this treatment, confirming is the negligible contribution of the substrate to the thermopower of GBCO films reported in this work. The similar order of magnitude of resistivity of LAO and LSAT materials precludes as well any contribution of those substrates to the thermopower of the films.

\section{Results and discussion}


Based on close matching criteria one could anticipate the alignment of $a$-, $b$ - and $c$-axes of GBCO with respect to the substrate and therefore the preferred growth orientations of the GBCO films. A rough estimate of the lattice mismatch is presented in Table I. To calculate the epitaxial strain induced by each substrate was calculated from

$$
\operatorname{mismatch}(\%)=-\frac{a_{b u l k}^{e f f}-a_{\text {film }}}{a_{b u l k}^{e f f}} \times 100
$$

using the pseudo-cubic (effective) in-plane lattice parameters of GBCO. The effective in-plane parameter is calculated as the geometric mean value of the two cell parameters within the matching plane depending on the crystal domain orientation, either $a_{\perp}, b_{\perp}$ or $c_{\perp}$-oriented. As can be seen in Table I, the mismatch difference between different orientations of GBCO is very large on STO so that the $c_{\perp}$-axis orientation, with a clear lower mismatch $(+0.18 \%)$, dominates. On the other hand, for the case of GBCO on LAO, the preferred growth direction is along $b_{\perp}$-axis (that is $c_{\|}$to the substrate), with a compressive strain $(-0.86 \%)$. In case of GBCO films on LSAT, an intermediate situation is foreseen where the most likely growth orientation can either be $c_{\perp}$-axis oriented (with a compressive strain of $-0.71 \%$ ), $a_{\perp}$-axis oriented (with a tensile strain of $+0.28 \%$ ) or a mix of both $c_{\perp}$ and $a_{\perp}$ orientations, since they show similar matching values. (although $b_{\perp}$ orientation could not be a priori discarded). Based on this calculation, the expected orientations of GBCO films on the different substrates are illustrated in Fig. 1d-f., as predominantly $c_{\perp}$ on STO, mixed $c_{\perp}, a_{\perp}, b_{\perp}$ (equally expressed as $c_{\perp}+c_{\|}$) on LSAT, and $b_{\perp}$ (equally expressed as pure $c_{\|}$) on LAO. Note that on LAO $c_{\|}$can lay in two orthogonal in-plane directions as depicted in the scheme.

Table I Expected strains and most preferred growth directions of GBCO film on STO, LSAT and LAO substrates

\begin{tabular}{|cccccc|}
\hline $\begin{array}{c}\text { Growth } \\
\text { orientation }\end{array}$ & $\begin{array}{c}\text { Matching } \\
\text { plane }\end{array}$ & $\begin{array}{c}\text { Effective } \\
\text { (pseudo) in-plane } \\
\text { of GBCO }(\AA)\end{array}$ & $\begin{array}{c}\text { Mismatch in } \\
\text { STO }(\%) a=\end{array}$ & $\begin{array}{c}\text { Mismatch in } \\
\text { LSAT }(\%) a=\end{array}$ & $\begin{array}{c}\text { Mismatch in } \\
\text { LAO }(\%) a=\end{array}$ \\
$c \perp$ & $a-b$ & $3.898(\sqrt{a \times(b / 2)}$ & +0.18 & $3.870 \AA$ & $3.790 \AA$ \\
& & & & -0.71 & -2.76 \\
$a \perp$ & $b-c$ & 3.859( & +1.19 & +0.28 & -1.76 \\
& & $\sqrt{(b / 2) \times(c / 2)})$ & & &
\end{tabular}




\begin{tabular}{|cccccc|}
\hline$b \perp$ & $c-a$ & $3.823(\sqrt{(c / 2) \times a}$ & +2.14 & +1.22 & -0.86 \\
& & & \\
\hline
\end{tabular}

The existence of $\mathrm{Gd} / \mathrm{Ba}$ cation ordering along $c$-axis forming a stack sequence of $-\left[\mathrm{GdO}-\mathrm{CoO}_{2}-\mathrm{BaO}-\right.$ $\mathrm{CoO}_{2}$ ]- layers (see Fig.1a) doubles the cell parameter from a simple perovskite, which is readily observed by the appearance of clear half-order $h k l / 2$ reflections in X-ray and electron diffraction experiments (halforder meaning with respect to main reflections of a primitive cubic perovskite). Therefore, cation ordering provides a way to identify the orientation of the $c$-axis. For example, for $c_{\perp}$-axis oriented GBCO crystal domains, the cation ordering can be found perpendicular to the film surface (Fig. 1a) while for $c_{\|}$-axis oriented GBCO crystal domains, the cation ordering should be parallel to the surface (Fig. 1a). Doubling along $b$-axis is related to oxygen vacancy ordering and is more difficult to observe since it involves lighter oxygen ions with lower scattering factor for X-rays.

(a)

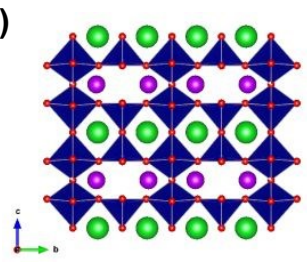

(b)

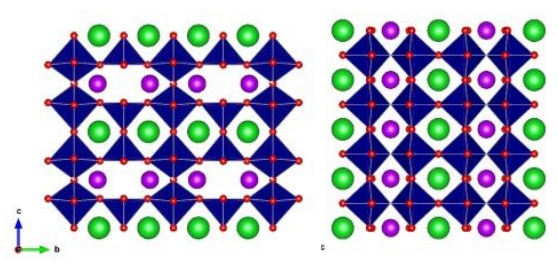

(c)

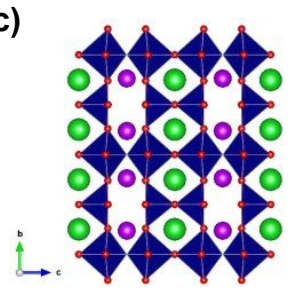

(d)


$3.934 \AA$ (e)

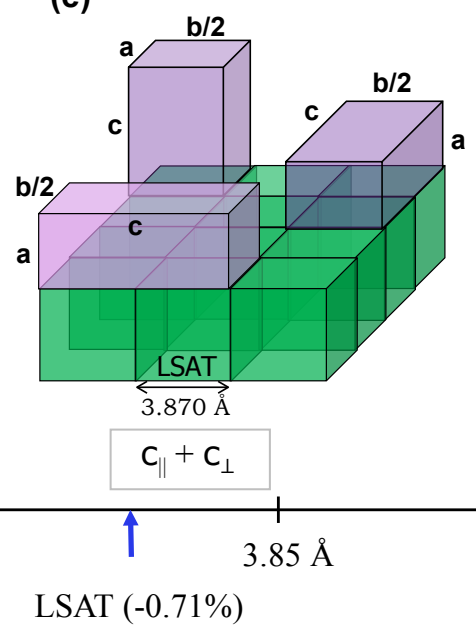

(f)

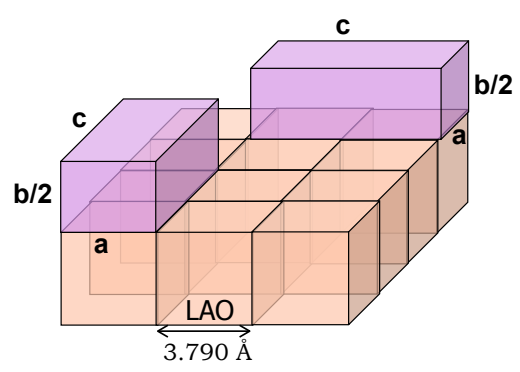

$\mathrm{b}_{\perp}\left(\& \mathrm{c}_{\|}\right)$

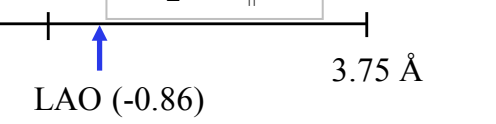

Fig. 1 Crystal structure and orientation of $\mathrm{GdBaCo}_{2} \mathrm{O}_{5.5}(\mathrm{GBCO})$ thin films growth on different substrates. a-c) Crystal structure of GBCO depending on the growth directions. Structure of GBCO is orthorhombic 
at room temperature, where $\mathrm{CoO}_{6}$ octahedrons and $\mathrm{CoO}_{5}$ pyramidal sites are in equal in number in stoichiometric composition. $\mathrm{GdO}$ and $\mathrm{BaO}$ layers are stacked alternatingly along crystallographic c-axis. d-f) Schematic illustration of the expected orientations of GBCO epitaxial films on STO, LSAT and LAO substrates according to the lattice mismatch calculation (see text). Bottom graph represents the substrates lattice parameters with expected lattice mismatch.

The film growth and crystal quality was studied through HRTEM analysis of several cross-section lamellae cut along one of the main [100] directions of the pseudo-cubic substrate. For $40 \mathrm{~nm}$ thick GBCO/STO (Fig. 2a), a clear contrast between horizontal rows with high and low brightness dots forming a sequence every two perovskite blocks along the vertical direction is observed. This is an indication of perovskite cell doubling along crystallographic $c$-axis, which corresponds to the alternate $\mathrm{GdO}$ and $\mathrm{BaO}$ sequence. This is further evident from the appearance of half order reflections parallel to [001]*, (vertical direction) marked with arrows, in the selected area electron diffraction (SAED) as shown in Fig 2b, which further demonstrates pure $c_{\perp}$-axis orientation.

Fig. 2c depicts the HRTEM image of GBCO/LSAT in [100] zone axis. A clear contrast in the bright and dark dots is visible in both horizontal and vertical direction, which indicates the coexistence of domains with different crystallographic $c_{\|}$and $c_{\perp}$ orientations. This image also shows other regions without doubling. These could either correspond to a single perovskite or more likely to double perovskite domains with $c_{\|}$axis orientation perpendicular to the plane of the image. There are not sharp grain boundaries between domains. Instead, there is a compact arrangement of the domains of different orientations. Therefore, the domains of mixed orientations seem to be strained in all directions. The cation ordering is further evident from the SAED image (Fig. 2d), where the half order-reflections can be observed in both horizontal and vertical direction.

Fig. 2e corresponds to the cross-section HRTEM of GBCO/LAO. It shows perfectly epitaxial arrangement. In this case, regions with doubling and no doubling were observed. A clear contrast in the bright and dark spots is visible in the horizontal direction. Domains with no double periodicity can be referred to as the domains with $c_{\|}$-axis perpendicular to the plane of image. This is also evident from SAED (Fig. 2f), which indicates that all domains show $c_{\|}$-axis parallel to the surface of substrate, either parallel or perpendicular to the cross-section image. No trace of the $c_{\perp}$-axis domains was observed in this case. 
Therefore, the HRTEM and SAED study of the GBCO films on different substrates provide an experimental validation of the preferred growth directions expected from simple lattice mismatch calculations on Table I.


Fig. 2 Nanoscale characterization of $\mathrm{GdBaCO}_{2} \mathrm{O}_{5.5}$ (GBCO) thin films. High-resolution transmission electron microscopy (HRTEM) observation of $40 \mathrm{~nm}$ thick GBCO films cross-sections. a) Perfect epitaxial arrangement of the GBCO film with STO substrates. Perovskite doubling is evident from the clear contrast between sequential horizontal atomic rows along the vertical direction indicating a pure $c_{\perp}$-orientation. $\mathrm{b}$ ) Perovskite doubling of GBCO/STO is also clear in the selected area electron diffraction (SAED) pattern with half order reflections indicated with arrows along the vertical [001]* direction. c) Perovskite doubling in GBCO/LSAT in parallel and perpendicular directions to the substrate was observed. Some domains are perpendicular to the plane of the paper. d) Half order reflections in parallel and perpendicular directions are also evident from SAED pattern. e) Cross-section HRTEM of GBCO/LAO film. Film is also perfectly epitaxial. A region with clear doubling is observed showing a contrast between bright and dark atomic columns along the horizontal direction (right part of the image). A region with no doubling is also observed 
(left part of the image) corresponding to $c$-axis perpendicular to the image. f) $c$-axis doubling parallel to the substrate surface is also evident from SAED indicating pure $c_{\|}$- orientation of the film.

In order to further study the crystallographic orientations and relative strains in the thin films, we performed X-ray diffraction (XRD) and high-resolution reciprocal space mapping (RSM). Fig. 3a depicts the standard $2 \theta-\omega$ scans of $25( \pm 2)$ nm of the GBCO films grown on STO, LSAT and LAO substrates. The XRD patterns of GBCO/STO shows only reflections, which can be indexed to $00 l$ indices, which indicates a growth orientation along $c_{\perp}$-axis. The double perovskite nature of the films i.e., the cation ordering, is evident from the appearance of both odd- (half-order reflections for a primitive cubic perovskite) and even symmetric $00 l$ reflections. This result is in agreement with the HRTEM and SAED observations. Reciprocal space map (RSM) around -303 reflection (Fig. 3b) shows that the film is fully strained in-plane with the STO substrate. The calculated in-plane and out-of-plane parameters are 3.905 and $3.780 \AA$ Á, respectively. This produces an average $+0.18 \%$ tensile in-plane strain and $-0.15 \%$ compressive strain along out-of-plane direction, with an overall $+0.21 \%$ increase in unit cell volume (see Table S2, in ESI).

The XRD pattern of GBCO/LSAT shows (Fig. 3a) the odd $00 l$ reflections from film, while the even $00 l$ reflections overlap with substrate reflections. On the other hand, RSM around -303 reflections (Fig. 3c) shows that the film reflections almost fully overlap with the substrate reflection. Thus, the in-plane and outof-plane cell parameters of GBCO film seem to be very close to that of LSAT, forming an almost cubiclike unit cell (cell parameters $a, b / 2, c / 2 \sim 3.870 \AA$ ). The macroscopic average cell parameters obtained by XRD are in agreement with the microscopic HRTEM observation (see Fig. 2c) where the domains of mixed orientations are locally strained in all directions to match to each other. The compact arrangement of domains of different orientations results in $+0.76 \%$ increase of the unit cell volume (see Table S3, ESI).

The standard $2 \theta-\omega$ pattern of GBCO/LAO (Fig. 3a) shows the appearance of peaks at similar positions of odd- and even reflections of $c_{\perp}$, as in the GBCO/STO case, which is contrary to the HRTEM and SAED study (Fig. 2e-f), where pure $c_{\|}$domains were observed. If one assumes that the XRD average measurement corresponds to $c_{\perp}$-domains the calculated in-plane strain on LAO would be $-2.8 \%$ (average between $a$ and $b / 2$ ), which is surprisingly large. The lack of observation of $c_{\perp}$-axis oriented domains in the HRTEM crosssections points instead towards $b_{\perp}$-axis orientation, with full in-plane matching with LAO substrate, as inferred from the RSM in Fig. 3d. This corresponds to an average in-plane compressive strain of $-0.88 \%$, and $-0.89 \%$ reduction of the unit cell volume (see details in Table S4, ESI). The observation of odd reflections in the perpendicular direction should then come from the fact that the structure is submitted to a large compressive strain, and could be accompanied by important structural changes with the 
displacement of the cation positions, so that the $b$-axis doubling is considerably enhanced and could be observed by XRD. In fact, the measured out-of-plane parameter (3.9687 A, see Fig. 3e) is much larger than bulk $b / 2(=3.934 \AA)$ parameter.
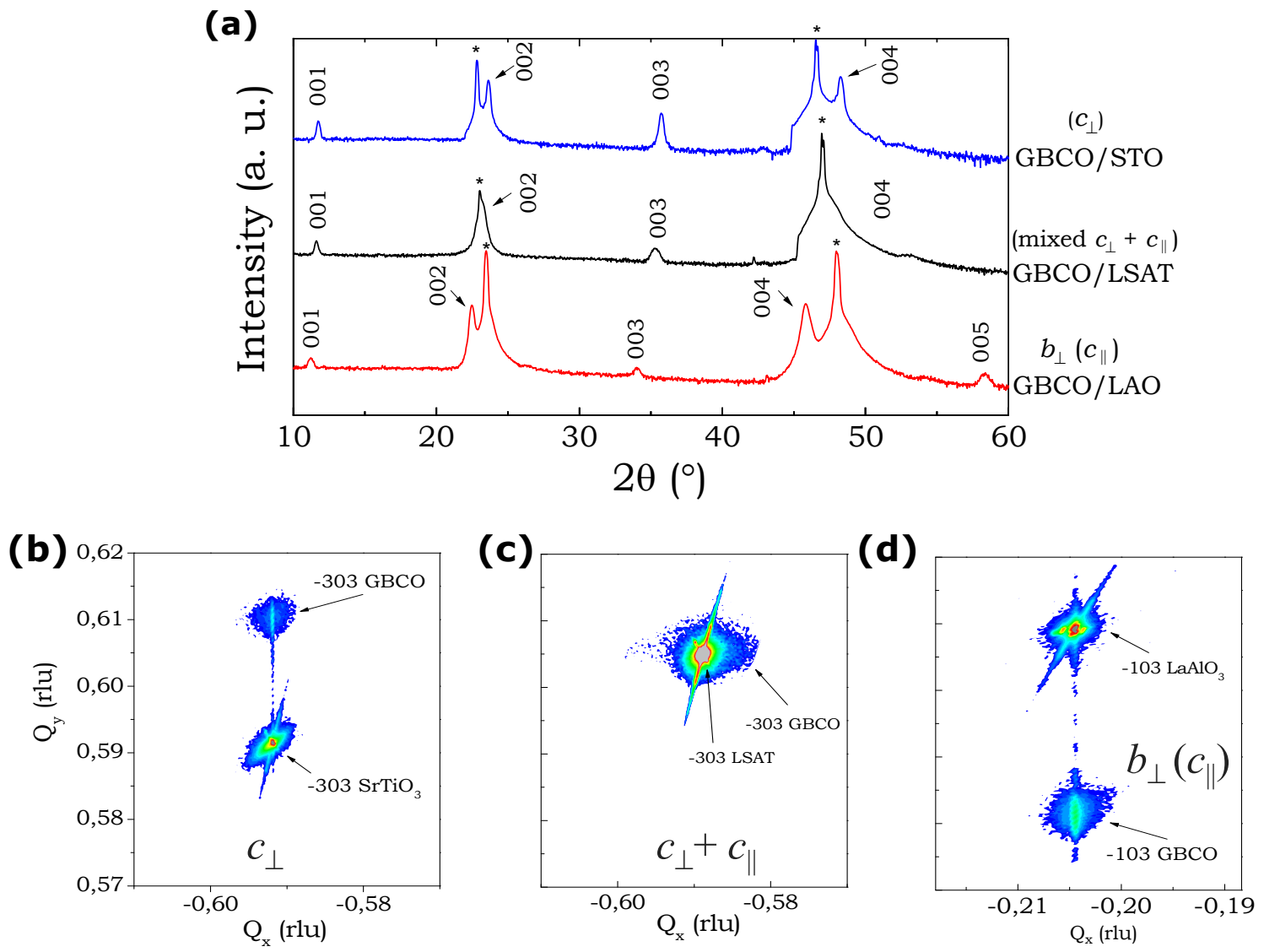

(e)

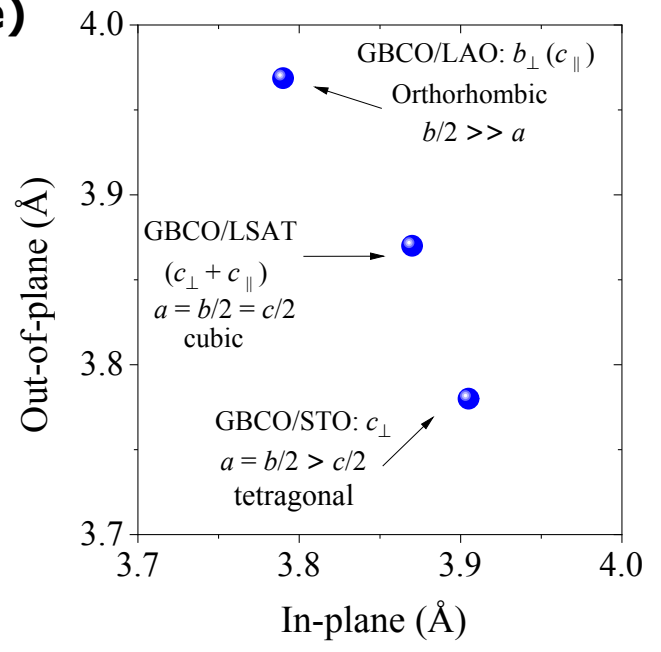

(f)

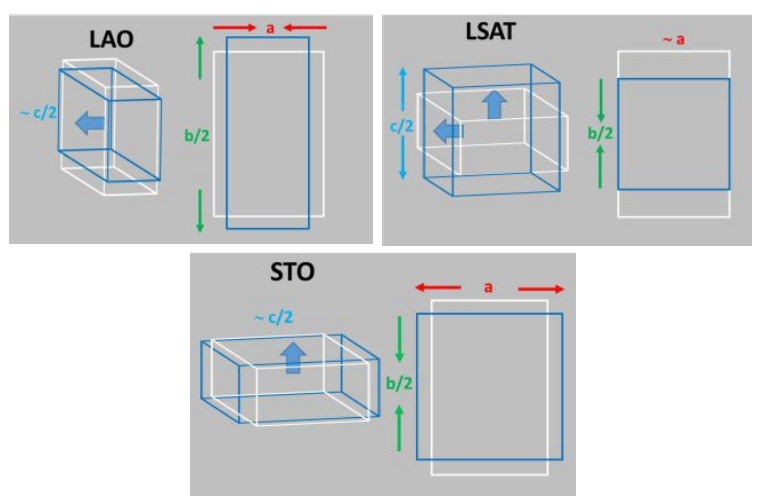


Fig. 3 X-ray characterization of GBCO films with same thickness (25 nm) grown on 001 oriented STO,


reflections were observed for all films. b-d) X-ray RSMs around -303, -303 and -103 reflections of STO, LSAT and LAO substrates respectively. e) Measured in-plane and out-of-plane cell parameters from the RSMs. f) The sketches show a 3D view and $a-b$ plane of GBCO domains indicating the distortions (blue lines) with respect to bulk GBCO (white lines). The blue arrows correspond to the direction of the $c$-axis. Note that in-plane matching on STO turns GBCO into a tetragonal structure, while it is almost cubic on LSAT and orthorhombic on LAO.

Figure 3e-f depict the in-plane and out-of-plane cell parameters measured from the RSMs and sketches the cell distortions obtained on the three different substrates, respectively. In summary, on STO, GBCO domains grow $c_{\perp}$-oriented with an in-plane tetragonal structure ( $a$ parameter expands while $b$ shortens, so $a=b / 2>c / 2$ ) producing an overall in-plane tensile strain. On LSAT, the structure is pseudo-cubic with mixed oriented domains, $a=b / 2=c / 2$; while on LAO, the overall in-plane compression of the $a-c$ plane produces an extension of the $b_{\perp}$-axis in the out-of-plane direction, with $b / 2>>a, c / 2$, as well as a larger orthorhombic distortion of the $a-b$ plane.

(a)

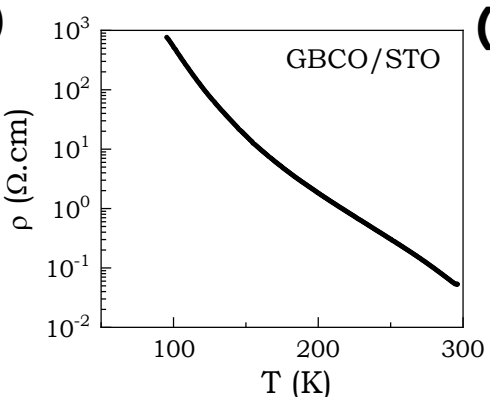

(d)

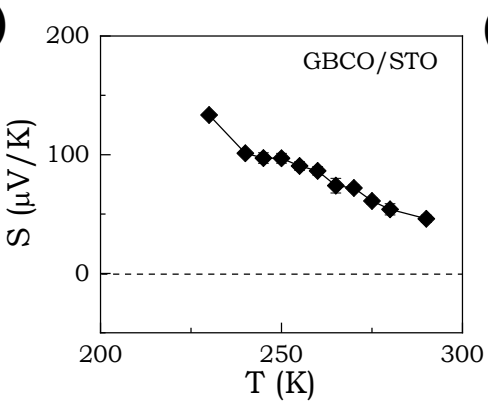

(b)

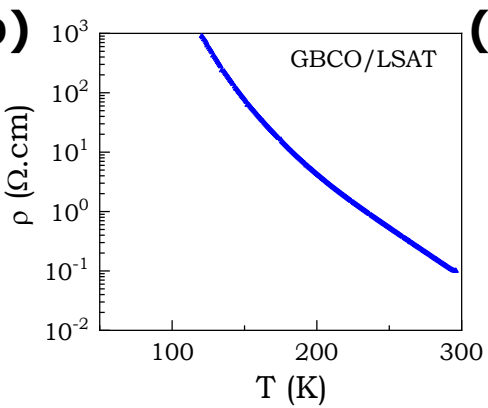

(c)



(f)
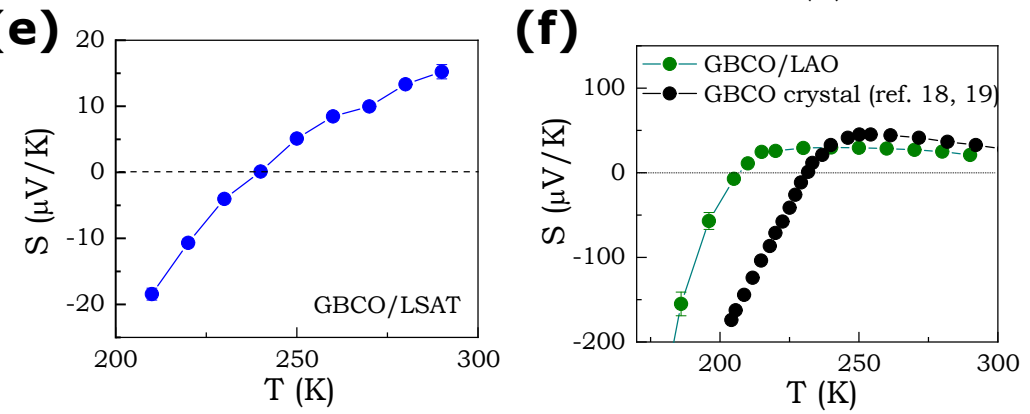

Fig. 4 Thermoelectric transport properties of GBCO thin films grown on STO, LSAT and LAO substrates. a-c) Temperature dependence of electrical resistivity of $25( \pm 2) \mathrm{nm}$ GBCO/STO, GBCO/LSAT and GBCO/LAO films, respectively. d-f) Temperature dependence of thermopower of GBCO/STO, 
GBCO/LSAT and GBCO/LAO films, respectively. GBCO/STO films shows positive thermopower throughout the temperature range of measurement, while GBCO/LSAT and GBCO/LAO shows change of sign of thermopower from negative to a positive one with increasing temperature.

The electrical resistivity $(\rho)$ and Seebeck coefficient $(S)$ of these $25 \mathrm{~nm}( \pm 2)$ GBCO films were measured at different temperatures as depicted in Fig. 4. Concerning resistivity, films showed similar magnitude and thermally activated behavior i.e., the $\rho$ dropped exponentially with increasing temperature from 100 to $300 \mathrm{~K}$ (see Fig. $4 \mathrm{a}-\mathrm{c}$ ). The drop of $\rho$ is almost five-order of magnitude: from $10^{3}$ to $5 \times 10^{-2}$ $\Omega . \mathrm{cm}$ in this temperature range. Surprisingly, despite the fact that the films correspond to different orientations, either $c_{\perp^{-}}$, mixed-oriented, or $b_{\perp^{-}}\left(\right.$or $\left.c_{\|^{-}}\right)$oriented, on STO, LSAT and LAO, respectively, their in-plane $\rho$ did not show any remarkable difference neither in magnitude nor in their temperature dependence, which is an indication of the poor intrinsic anisotropy and null role of grain boundaries in charge transport.

Although, it showed thermal activation-like behavior, the whole range of $\rho$ - $T$ cannot be fit either with Arrhenius model or small polaron hopping model (see Fig. S2a-b, ESI). Better fits were obtained when variable range hopping $(\mathrm{VRH})$ model was implemented ${ }^{23}$ and dimension of the VRH hopping varied from 3.2 to 3.8 (see Fig. S3, ESI). This type of conduction is typically exhibited by disordered systems, where charge carriers move by hoping between localized electronic states. However, no conclusive information about the conduction mechanism of charge carriers can be drawn from the $\rho$ - $T$ curve.

However, very different behavior is observed in the Seebeck coefficient, as shown in Fig. 4 d-f. GBCO/STO (Fig. 4d) shows large and positive $S=+138 \mu \mathrm{V} / \mathrm{K}$ at $230 \mathrm{~K}$, decreasing with temperature, as expected for a $p$-type semiconductor.

On the other hand, GBCO/LSAT (Fig. 4e) showed a negative $S=-20 \mu \mathrm{V} / \mathrm{K}$ at $210 \mathrm{~K}$, which increases with temperature and crosses to positive values at $\mathrm{T}=240 \mathrm{~K}$, and further increases up to $S \sim+15 \mu \mathrm{V} / \mathrm{K}$ at $290 \mathrm{~K}$.

Finally, GBCO/LAO (Fig. 4f) showed a large negative $S \sim-150 \mu \mathrm{V} / \mathrm{K}$ at $180 \mathrm{~K}$, which increased steeply to almost zero when temperature increased from 180 to $210 \mathrm{~K}$. The $S$ remained almost temperature independent from 220 to $290 \mathrm{~K}$, close to $\approx+25 \mu \mathrm{V} / \mathrm{K}$. This dependence is very similar to that reported for single-crystal GBCO with a slight oxygen deficiency ( turning the material into $n$-type with $\approx 4 \%$ of excess electrons) ${ }^{18,19,24}$, reaching a comparable constant value at high temperatures (values also included in Fig. 4f). 
Such variation of $S$, in sign and temperature dependence, for the different cases while maintaining a constant resistivity is quite distinct to that of a typical semiconducting (or band gap) material where $\rho(\mathrm{T})$ and $S(\mathrm{~T})$ are expected to follow a similar trend, and indicates the more subtle nature of electron-phonon interactions in transition metal oxides.

A complete explanation of the Seebeck coefficient dependence would require a deep knowledge of the Co ions spin distribution and magnetic phases for such complex Co-oxide, already described for bulk material $^{19}$, but not known for strained structures. A qualitative comparison with $\rho$ and $S$ dependence reported in $\mathrm{GdBaCO}_{2} \mathrm{O}_{5.5 \pm \delta}$ single crystals ${ }^{19}$ indicate some similarities. In bulk crystals at low temperatures $(T=100 \mathrm{~K})$ a very small deviation in stoichiometry from nominal $\delta=0$ causes a huge change in $S$, from about $S \sim-700 \mu \mathrm{V} / \mathrm{K}$ at $\delta=-0.01$, to $S \sim+400-600 \mu \mathrm{V} / \mathrm{K}$ at $\delta=+0.01$. While resistivity at $T=100 \mathrm{~K}$ varies less than one order of magnitude in this stoichiometry range $\delta= \pm 0.01$, and becomes even less pronounced at higher temperatures. In this sense the variations observed in our films on different substrates point towards the same trend of a major contribution to the Seebeck coefficient variations due to subtle changes in film oxygen stoichiometry. The film on STO, which showed a large positive $S$ value, would then correspond to $p$-type $\mathrm{GBCO}$ with a stoichiometry $5.5+\delta$, with a certain concentration of $\mathrm{Co}^{4+}$ ions from nominal $\mathrm{Co}^{3+}$ at $\delta=0$, while on LAO it would correspond to $5.5-\delta$, with a concentration $\mathrm{Co}^{2+}$ ions. The LSAT case with a small negative $S$ value at low temperatures may correspond to an intermediary situation with $\delta$ close to zero, close to nominal $\mathrm{Co}^{3+}$. At this point, it is not yet possible to offer a quantitative measure of $\delta$ values.

To support this correlation the behavior of Seebeck coefficient in the so-called "high temperature" limit offers some insights to the explanation of the observed differences.

At high temperature, the thermoelectric power of the films approach (or can be extrapolated to) a fairly constant value. In this regime, thermopower is expected to be governed by the statistical distribution of charge carriers over available sites i.e., the configurational entropy. The magnitude of $S$ in this limit is typically quantified by the Heike's equation ${ }^{25}$ :

$$
S=-\frac{k_{B}}{e} \ln \left(\frac{x}{1-x}\right)
$$

with $x$ being the concentration of holes and $\kappa_{B}$ and e are Boltzmann constant and elementary charge of electron, respectively. GBCO film on LAO (submitted to compressive strain) shows at high- $T$ a value of $S$ $\approx+23 \mu \mathrm{V} / \mathrm{K}$, which could be attributed to an oxygen deficiency $\left(\mathrm{GdBaCo}_{2} \mathrm{O}_{5.5-\delta}, \delta \approx 0.08\right)$. For single-crystal GBCO $S \approx+13 \mu \mathrm{V} / \mathrm{K}$ (values included in Fig. 4f along with film on LAO), which has been reported to correspond to a slight oxygen deficiency $\left(\mathrm{GdBaCo}_{2} \mathrm{O}_{5.5-\delta}, \delta \approx 0.04\right) .{ }^{18,19,24}$ For GBCO/LSAT, there is also a 
crossing from negative to positive value, but $S$ did not yet reach the constant temperature independent value at the maximum temperature of the measurement (room temperature), but a rough extrapolation predicts that it will stabilize at positive values, around $+20-30 \mu \mathrm{V} / \mathrm{K}$ at high enough temperature, similar to that on LAO, so a slight oxygen deficiency is also foreseen in this case.

Similarly, for GBCO/STO, $S$ is still far from being constant at room temperature, but again, fitting the data to an exponential function predicts a $S \approx 0$ or slightly negative at sufficiently high temperature, pointing towards a very slight oxygen excess, leading to hole conduction.

Therefore, both low and high temperature $S$ values in GBCO epitaxial thin films grown on different substrates seem to indicate that the most probable contribution to the observed variations corresponds to a slight change in oxygen stoichiometry.

Although, a possible cation composition deviation cannot be ruled out. For this purpose, EELS analysis was performed on the film cross sections (Fig. S4. ESI). Unfortunately, the overlap between Co $L$ and Ba $M$ lines prevented to carry out any quantitative analysis of $\mathrm{Ba} / \mathrm{Co}$ composition, as well as of the Co oxidation state. ${ }^{26,27,28}$ Qualitatively, there are no substantial differences in the EELS profiles between films, which indicates that there are no large deviations in cation composition. EDX experiments (see Fig. S5 and Table $\mathrm{S} 1$, ESI), on the other hand, also suggest that $\mathrm{Gd} / \mathrm{Ba}$ ratio is constant for all the films and, only a slightly lower amount of Co is observed in GBCO/STO film.

It is already well established that spontaneous generation of oxygen vacancies can occur in transition metal oxide thin films as a mechanism to accommodate the epitaxial strain imposed by the substrate..$^{29,30} \mathrm{In}$ standard perovskites with diluted and disordered oxygen vacancies a tensile strain reduces the oxygen vacancy formation energy to accommodate a larger concentration of oxygen vacancies. ${ }^{30}$ This results from the change in the oxidation state of transition metal oxide, increasing its cation radius, which makes the cell volume to expand when increasing oxygen vacancy concentration. ${ }^{31}$ However, $\mathrm{GdBaCo}_{2} \mathrm{O}_{5.5 \pm \delta}$ behaves in an opposed way and increases cell volume when increasing oxygen content, ${ }^{32}$ resulting from a complex compensation of the expansions of individual $\mathrm{BaCo}_{2} \mathrm{O}_{5}$ and $\mathrm{GdO}_{\delta}$ blocks in the structure. ${ }^{33}$ If we look at the cell volume changes in the deposited films with respect to reported bulk structure for $\mathrm{GdBaCo}_{2} \mathrm{O}_{5.5}$ nominal stoichiometry (Tables S2, S3 and S4, ESI) there is a $+0.21 \%$ increase on STO, $+0.76 \%$ on LSAT, and $-0.89 \%$ reduction on LAO. Concentrating in the films with a dominant single orientation, $c_{\perp}$ on STO and $c_{/ /}$on LAO, the cell volume would induce an increase $(\delta>0)$ or a decrease $(\delta<0)$ in oxygen concentration from nominal $\mathrm{GBCO}_{5.5}$, which is consistent with the positive and negative $S$ values measured for those films. 
Following the same reasoning, on LSAT the large cell volume increase (in fact almost four times larger than on STO) would induce a positive $S$ value, while the measurement indicates a negative value. Whether there are additional contributions to the $S$ dependence related to the differences in microstructure cannot be ruled out in this study. Note that pure $c_{\perp}$-oriented films grown on STO do not show important grain boundaries, other than possible mixed $a_{\|}$and $b_{\|}$in the plane of the sample, while both mixed $\left(c_{\perp}+c_{\|}\right)$oriented films (on LSAT) and pure $c_{\|}$-oriented films (on LAO) have domains where the corresponding $c$ axes form $90^{\circ}$. As previously observed in the comparison of films resistivity the electronic transport through these boundaries does not affect the overall behavior. However, phonon transport, which is known in general to be considerably affected by the presence of boundaries, may contribute to the changes in $S$ coefficient, particularly in the film on LSAT. Although this fact is not unambiguously proven in this study, it deserves a further study but was out from the scope of the present work.

Another possible explanation for the changes in oxygen stoichiometry of the films could be related to the distortions in the $a-b$ plane of the GBCO structure induced by strain, i.e. the degree of orthorhombicity. When looking at Fig. $3 \mathrm{f}$, one observes that $a-b$ plane in $c_{\perp}$ - oriented film on STO is strained to a tetragonal structure with $a=b / 2$, while on LAO the distortion goes in the sense of increasing the difference between $a$ and $b / 2$. In the reported $a$ and $b / 2$ values for single crystal $\mathrm{GBCO}_{5.5 \pm \delta}$ for different oxygen stoichiometry ${ }^{24}$ the value of $b / 2$ reduces with increasing $\delta$, while $a$ increases, in the vicinity of the nominal stoichiometry $\delta=0$, within the region of orthorhombic Pmmm structure. That means the difference between $a$ and $b / 2$ is reduced when increasing oxygen content. This will agree with our assumption that oxygen content increases on STO, while reduces on LAO, in agreement with the observed variations in the sign of $S$ coefficient at low temperatures. Again, on LSAT the film domains presumably stained to a cubic structure, will increase oxygen stoichiometry resulting in a positive $\mathrm{S}$ coefficient, contrary to the observed negative value. As commented before in this case the complex microstructure and large local strains could results in a completely different situation.

It is clear from the above discussion that GBCO films on different substrates have a variety of tunable parameters such as films orientation, strain, microstructure, spin states of cobalt ions, and oxygen vacancies. All of these parameters might influence the thermoelectric properties of thin films and hence, the interpretation on the origin of variable thermopower of GBCO films is a delicate issue. However, based on the experimental evidences in this work, we argue that the anisotropy of the charge transport properties is not significant, while the subtle variations of oxygen stoichiometry accompanying film strain lead to the variation of the temperature dependence and sign of thermopower. 


\section{Summary}

In summary, control on growth orientation of high quality GBCO thin films was achieved by epitaxial strain engineering. The thermoelectric power in these systems shows a change from $p$-to- $n$ type depending on whether a compressive or a tensile stress is applied, which is related to the adapting oxygen stoichiometry to partially release the strain imposed by the substrate. This is an important result from the point of view of thermoelectric properties, as $n / p$ type response can be attained in the same material by adjusting the substrate mismatch. We propose that the spontaneous generation of oxygen vacancies to accommodate cell volume changes, as well as the particular orthorhombicity of $a-b$ plane, is responsible of this interesting behavior.

\section{Acknowledgements}

This work was supported from the projects: MINECO ref. MAT2016-77100-C2-1-P; CNRS-CSIC PICS Project ref.261091, the EU for funding through project H2020-MSCA-RISE-2014 ref.645658, and the AGAUR agency for 2017SGR327. ICN2 is funded by the CERCA programme/Generalitat de Catalunya and by the Severo Ochoa programme of the Spanish Ministry of Economy, Industry and Competitiveness (MINECO, grant no. SEV-2017-0706). CMS and ECA acknowledge support from Spanish Ministry MINECO/FEDER: FIS2015-70862-P PHENTOM. AC particularly acknowledges the support of Severo Ochoa programme and ICN2 for his PhD grant. FR acknowledges support from MAT2016-80762-R, Xunta de Galicia (Centro singular de investigación de Galicia accreditation 2016-2019, ED431G/09), by the European Union (European Regional Development Fund-ERDF) and the European Commission through the Horizon H2020 funding by H2020-MSCA-RISE-2016-Project No. 734187-SPICOLOST

\section{References}

1 J. R. Sootsman, D. Y. Chung and M. G. Kanatzidis, Angew. Chemie - Int. Ed., 2009, 48, 86168639 .

2 I. Terasaki, J. Appl. Phys., 2011, 110, 053705.

3 I. Terasaki, APL Mater., 2016, 4, 104501.

4 I. Terasaki, Y. Sasago and K. Uchinokura, Phys. Rev. B, 1997, 56, R12685-R12687.

5 M. Lee, L. Viciu, L. U. Li, Y. Wang, M. L. Foo, S. Watauchi, R. A. P. Jr, R. J. Cava and N. P. Ong, Nat. Mater., 2006, 5, 537-540.

6 R. Funahashi and M. Shikano, Appl. Phys. Lett., 2002, 81, 1459-1461. 

111, 103709.

M. Shikano and R. Funahashi, Appl. Phys. Lett., 2003, 82, 1851-1853.

T. Tsubota, M. Ohtaki, K. Eguchi and H. Arai, J. Mater. Chem., 1997, 7, 85-90.

T. Okuda, K. Nakanishi, S. Miyasaka and Y. Tokura, Phys. Rev. B - Condens. Matter Mater. Phys., 2001, 63, 113104.

11 S. Ohta, T. Nomura, H. Ohta and K. Koumoto, J. Appl. Phys., 2005, 97, 034106.

12 Y. Wang, Y. Sui, H. Fan, X. Wang, Y. Su, W. Su and X. Liu, Chem. Mater., 2009, 21, 4653-4660.

13 W. Koshibae, K. Tsutsui and S. Maekawa, Phys. Rev. B, 2000, 62, 6869-6872.

14 W. Koshibae and S. Maekawa, Phys. Rev. Lett., 2001, 87, 236603.

15 H. J. Xiang and D. J. Singh, Phys. Rev. B, 2007, 76, 195111.

16 D. J. Singh and D. Kasinathan, J. Electron. Mater., 2007, 36, 736-739.

17 D. J. Singh, Phys. Rev. B, 2000, 61, 397-402.

18 A. A. Taskin, A. N. Lavrov and Y. Ando, Phys. Rev. B, 2006, 73, 0121101 (R).

19 A. A. Taskin and Y. Ando, Phys. Rev. Lett., 2005, 95, 176603.

20 X. F. Sun, A. A. Taskin, X. Zhao, A. N. Lavrov and Y. Ando, Phys. Rev. B - Condens. Matter Mater. Phys., 2008, 77, 054436.

21 A. A. Taskin, A. N. Lavrov and Y. Ando, Phys. Rev. Lett., 2003, 90, 227201.

22 C. Frontera, J. L. García-Muñoz, A. Llobet and M. A. G. Aranda, Phys. Rev. B, 2002, 65, 180405.

23 Y. Machida, X. Lin, W. Kang, K. Izawa and K. Behnia, Phys. Rev. Lett., 2016, 116, 087003.

24 A. A. Taskin, A. N. Lavrov and Y. Ando, Phys. Rev. B - Condens. Matter Mater. Phys., 2005, 71, 134414.

25 P. M. Chaikin and G.Beni, Phys. Rev. B, 1976, 13, 647.

26 D. H. Pearson, B. Fultz and C. C. Ahn, Appl. Phys. Lett., 1988, 53, 1405-1407.

27 D. H. Pearson, C. C. Ahn and B. Fultz, Phys. Rev. B, 1993, 47, 8471-8478. 
29 Y. Y. Zhang, R. Mishra, T. J. Pennycook, A. Y. Borisevich, S. J. Pennycook and S. T. Pantelides, Adv. Mater. Interfaces, 2015, 2, 1500344.

30 U. Aschauer, R. Pfenninger, S. M. Selbach, T. Grande and N. A. Spaldin, Phys. Rev. B - Condens. Matter Mater. Phys., 2013, 88, 054111.

31 D. Marrocchelli, N. H. Perry and S. R. Bishop, Phys. Chem. Chem. Phys., 2015, 17, 10028-10039.

32 A. Chatterjee, J. M. Caicedo, B. Ballesteros and J. Santiso, J. Mater. Chem. A, 2018, 6, 1243012439.

33 N. Ishizawa, T. Asaka, T. Kudo, K. Fukuda, A. Yasuhara, N. Abe and T. H. Arima, Chem. Mater., 2014, 26, 6503-6517. 

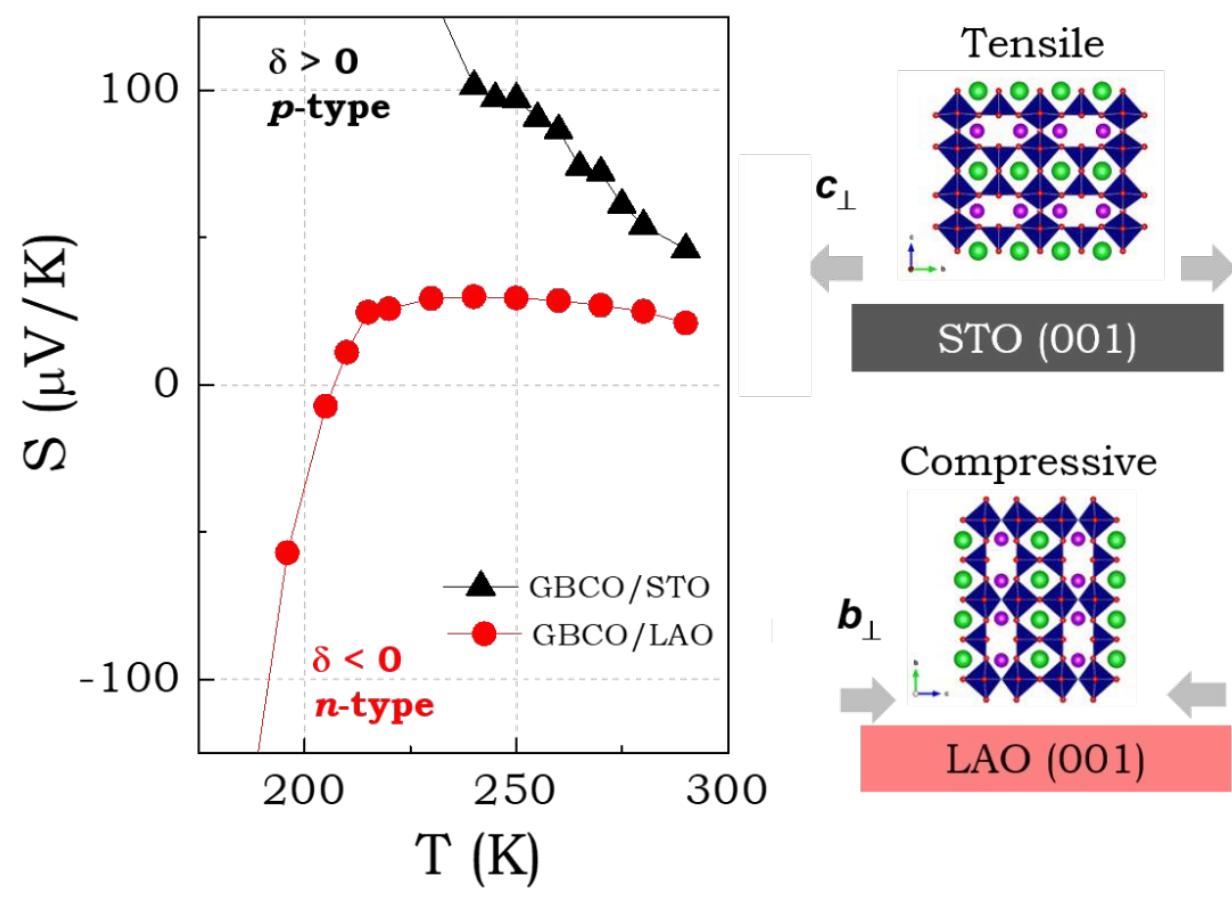

Oxygen stoichiometry, $\delta$, in epitaxial $\mathrm{GdBaCo}_{2} \mathrm{O}_{5.5 \pm \delta}$ thin films varies to accommodate the strain, which substantially affects its thermoelectric properties, bringing the material from $p$-type (tensile strain $c_{\perp}$ oriented on STO) to $n$-type thermopower (compressive strain $b_{\perp}$ on LAO). 